\title{
Succinate dehydrogenase and human diseases: new insights into a well-known enzyme
}

\author{
Pierre Rustin ${ }^{*, 1}$, Arnold Munnich ${ }^{1}$ and Agnès Rötig ${ }^{1}$ \\ ${ }^{1}$ Unité de Recherches sur les Handicaps Génétiques de l'Enfant (INSERM U393) Hôpital Necker-Enfants Malades, \\ 149, rue de Sèvres, Paris 75015, France
}

\begin{abstract}
Inherited defects of the mitochondrial succinate dehydrogenase (SDH) in humans are associated with striking variable clinical presentations ranging from early-onset devastating encephalomyopathy to tumour susceptibility in adulthood, or optic atrophy in the elderly. Although different genes encoding the four subunits of the SDH have been found mutated in association with these different phenotypes, we propose that the wide clinical spectrum actually originates from the specific roles of the SDH in the respiratory chain and the mitochondria. In particular, beside its function in the Krebs cycle and the respiratory chain, the specific redox properties of the enzyme could confer to the SDH a specific function in superoxide handling. European Journal of Human Genetics (2002) 10, 289 - 291. doi:10.1038/sj.ejhg.5200793
\end{abstract}

Keywords: succinate dehydrogenase; mitochondria; paraganglioma; phaeochromocytomas; encephalomyopathy

\section{Introduction}

The mitochondrial succinate dehydrogenase (SDH) complex catalyses the oxidation of succinate to fumarate in the Krebs cycle, and feeds electrons to the respiratory chain ubiquinone (UQ) pool ${ }^{1,2}$ (Figure 1). SDH consists of four nuclearly encoded subunits whose structure and genes being mostly conserved through evolution. In contrast, the other respiratory complexes have gained additional subunits, or conversely can be absent in some organisms, eg complex I in the yeast Saccharomyces cerevisiae. The subunits A and B form the SDH sensu stricto. The other two, subunits $\mathrm{C}$ and $\mathrm{D}$, binding a $b$-type cytochrome, are often referred to as the anchoring subunits, although they are actually required for electron transfer from succinate to the ubiquinone pool as well.

Defects of the succinate dehydrogenase are comparatively rare in human. ${ }^{3}$ Yet, large differences in clinical presenta-

*Correspondence: P Rustin, Unité de Recherches sur les Handicaps Génétiques de I'Enfant (INSERM U393) Hôpital Necker-Enfants Malades, 149 , rue de Sèvres, Paris 75015, France.

Tel: +33 14449 5161; Fax: +33 14734 8514; E-mail: rustin@necker.fr Received 19 November 2001; revised 4 February 2002; accepted 6 February 2002 tions have been reported in patients harbouring mutations in one of the four SDH subunit-encoding genes. Mutations in the flavoprotein subunit, SDHA, the very first mutations in a nuclear gene reported to cause a mitochondrial RC defect in mid '90s result in typical mitochondrial encephalopathy, namely Leigh syndrome. ${ }^{4,5}$ More recently, mutations in subunits $\mathrm{B}, \mathrm{C}$ and $\mathrm{D}$ have been shown to cause paraganglioma, generally benign, vascularised tumours in the head and the neck, or phaeochromocytomas. ${ }^{6-8}$ The carotid body, the most common tumour site, is a highly vascular small organ localised at the bifurcation of the common carotid artery and is a chemoreceptive organ that senses oxygen levels in the blood. Because of the occurrence of somatic mutations of these three genes in tumours, they should be regarded as tumour-suppressor genes. $^{9}$

So far, SDH is only known to catalyse a unique reaction, which requires the participation of its four subunits, and deleterious mutations in any of the SDH genes should invariably result in a decreased SDH activity. Therefore, the striking phenotypic differences associated with mutations in the four subunits raise puzzling questions. The re-investigation of the long-known properties of the SDH might help to shed light on this problem. 


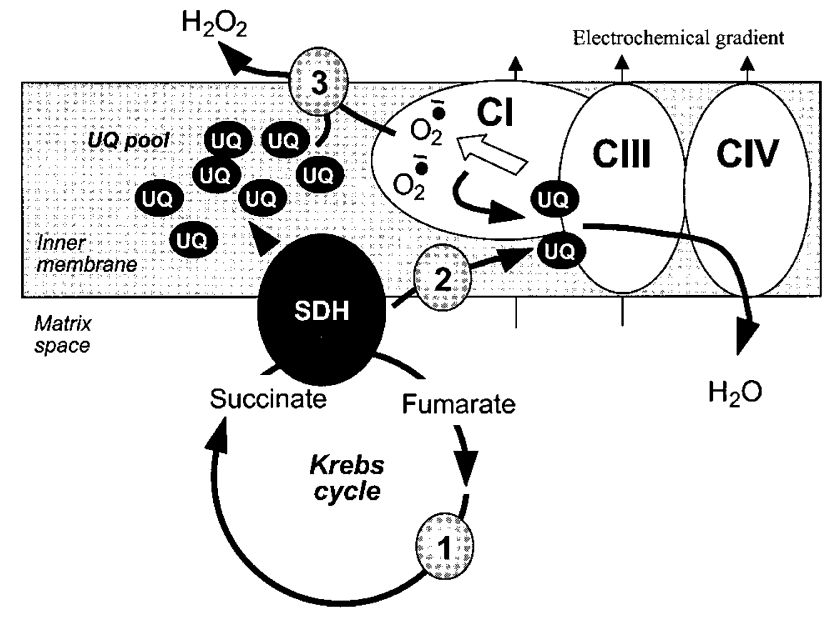

Figure 1 The several functions of the succinate dehydrogenase in the mitochondria. The succinate dehydrogenase catalyses the oxidation of succinate into fumarate in the Krebs cycle (1), derived electrons being fed to the respiratory chain complex III to reduce oxygen and form water (2). This builds up an electrochemical gradient across the mitochondrial inner membrane allowing for the synthesis of ATP. Alternatively, electrons can be diverted to reduce the ubiquinone pool (UQ pool) and provide reducing equivalents necessary to reduce superoxide anions originating either from an exogenous source or from the respiratory chain itself (3). A complete lack of succinate dehydrogenase activity will hamper electron flow to both respiratory chain complex III and the quinone pool, resulting in a major oxidative stress known to promote tumor formation in human. UQ: ubiquinone; $\mathrm{Cl}, \mathrm{CIII}, \mathrm{CIV}$ : the various complexes of the respiratory chain.

\section{The unique properties of the SDH and its partnership with the ubiquinone pool}

Contrasting with most dehydrogenases feeding electrons to the $\mathrm{RC}, \mathrm{SDH}$ is known to be fully activated upon reduction of the RC and in the presence of ATP, due to dissociation of its physiological inhibitor, oxaloacetate, at the active site. ${ }^{10}$ As a result, in the presence of ATP, the decreased electron flow from succinate to oxygen is still compatible with a high reduction of the UQ pool. Additionally, UQ concentration in the mitochondrial inner membrane, which is rapidly limiting for most substrate oxidation upon UQ extraction, is only poorly limiting for succinate oxidation. ${ }^{11}$ This suggests that the SDH might 'see' (requires less) UQ when other dehydrogenases do not. In keeping with this, oxygen uptake measured with isolated mitochondria in the presence of a combination of NADH and succinate is less than the sum of the individual rates, mainly because of the reduction of the electron flow from the NADH dehydrogenase. ${ }^{12}$ This again indicated the successful competition of the SDH to reduce the UQ pool. The powerful reducing activity of the SDH is also illustrated by its distinctive ability to possibly trigger reverse electron flow through the respiratory chain complex I, ultimately resulting in NADH production. Reverse electron flow from succinate to NADH has been reported in higher plants and mammalian cells. ${ }^{13-15}$ All these observations support the view that SDH plays a specific role in the maintenance of the mitochondrial UQ pool reduction.

Ubiquinone, beside its function in the RC as an electron carrier mediating electron transfer between the various dehydrogenases and the cytochrome path, is admittedly working as a powerful antioxidant in biological membranes. ${ }^{16}$ Possibly for this exact reason, it is in much larger amounts compared to other electron carriers of the $\mathrm{RC}^{1}$ including the sum of the dehydrogenases. Then, only a portion of the UQ pool may be actually involved in electron transfer depending on dehydrogenases involved. Accordingly, the measurable redox status of the UQ pool should result from the reducing activity of the different dehydrogenases, the oxidising activity of complex III and the kinetic equilibrium in the pool. The UQ pool therefore represents an electron sink and, when reduced, an antioxidant reservoir in the mitochondrial inner membrane. However, UQ is a double-faced compound, possibly working as either an antioxidant when fully reduced to ubiquinol, or a pro-oxidant when semi-reduced to the unstable ubisemiquinone form. ${ }^{16}$ Possibly together with reduced cytochrome $b$, semi-reduced quinones constitute the prominent source of superoxides by the RC under state 4 conditions. ${ }^{1}$ Finally, when defective, the RC can produce an abnormal amount of superoxides involving additional RC components, eg flavin radicals of complex I. ${ }^{17}$ Delivering electrons for the full reduction of UQ to $\mathrm{UQH}_{2}$ might then be of a tremendous importance for the control of oxygen toxicity in the mitochondria. Therefore, the $\mathrm{SDH}$, thanks to its unique redox properties, may be a key enzyme to control UQ pool redox poise under these conditions.

\section{SDH deficiencies: reduced electron flow and/or increased oxygen toxicity}

It is noticeable that inherited deficiencies of SDH associated with SDHA mutations are always associated with relatively high residual activities, ranging from $25-50 \%$ of control mean values. ${ }^{4,5}$ As a comparison, less than $5 \%$ residual activity is frequently measured in patients with severe defect of complex IV or I. However, patients with such SDH defect present typical Leigh syndrome and thus do not clinically differ from patients with other RC complex defects. ${ }^{18}$ Interestingly enough, a late-onset optic atrophy has also been ascribed to a heterozygous mutation in SDHA in two patients with $50 \%$ residual SDH activity. ${ }^{19}$ In none of these cases was the presence of tumour reported.

Contrasting with the high residual activity measured in patients with mutations in the SDHA gene, SDH activity is barely detectable in tumour tissues from patients with hereditary paraganglioma. ${ }^{20}$ The total lack of activity predictably originates from the highly deleterious mutations identified in the only allele expressed in the tumour. As 
discussed above, the lack of SDH activity will not only cause decreased ATP production-a condition that results from severe CI or CIV defect as well - but will also deprive the RC of the only dehydrogenase activity able to maintain a high reduction status of the UQ pool. This may in turn cause the loss of the anti-oxidant capacity of the respiratory chain and possibly an oxidative stress that is known to readily trigger tumour formation.

\section{Oxygen and SDH: worms as well}

Observations made with the worm Caenorhabditis elegans also support the idea that the SDH plays a specific role in the handling of oxygen by mitochondria. Strangely enough, a specific SDH mutation-rather than a whole class of respiratory chain mutants-was identified in an oxygenhypersensitivite worm. ${ }^{21}$ Tumour formation has not been described in C. elegans, but the mutant worm shows a shortened life span. In humans, mutations in any of the SDH cause the complex II to fully disassemble. Then, being absent, complex II can be disregarded as a source of additional superoxide production. Thus, we propose that the superoxide overproduction, admittedly leading to tumour formation in human and hypersensitivity to oxygen in the mutant worm, should be ascribed to the decreased ability of the SDH to adequately reduce the UQ pool, a necessary condition to resist oxidative stress.

Studying SDH-related human diseases therefore suggests that the enzyme not only plays a central role in the Krebs cycle and the respiratory chain, but also differs from other mitochondrial dehydrogenases thanks to its unique redox properties. In partnership with ubiquinone, SDH would represent a crucial antioxidant enzyme in the mitochondria controlling superoxides scavenging activity of the RC. It is therefore perhaps not so surprising that a wide spectrum of human diseases echoes the mutations in this multi-functional enzyme.

\section{References}

1 Tzagoloff A: Mitochondria. New York, Plenum Press, 1982, pp 1 342.

2 Ackrell BAC, Johnson MK, Gunsalus RP, Cecchini G: Structure and function of succinate dehydrogenase and fumarate reductase, in Muller F (ed): Chemistry and Biochemistry of Flavoproteins. Boca Raton, FL: CRC Press. 1992, vol III, pp 229-297.

3 Rustin P, Rötig A: Inborn errors of complex II-Unusual human mitochondrial diseases. Biochim Biophys Acta 2001; 1553: 117 122.
4 Bourgeron T, Rustin P, Chretien D, et al: Mutation of a nuclear succinate dehydrogenase gene results in mitochondrial respiratory chain deficiency. Nature Genet 1995; 11: 144-149.

5 Parfait B, Chretien D, Rötig A, Marsac C, Munnich A, Rustin P: Compound heterozygous mutations in the flavoprotein gene of the respiratory chain complex II in a patient with Leigh syndrome. Hum Genet 2000; 106: 236-243.

6 Baysal BE, Ferrell RE, Willett-Brozick JE et al: Mutations in SDHD, a mitochondrial complex II gene, in hereditary paraganglioma. Science 2000; 287: 848-851.

7 Niemann S, Muller U: Mutations in SDHC cause autosomal dominant paraganglioma, type 3. Nature Genet 2000; 26: 268 270.

8 Astuti D, Latif F, Dallol A et al: Gene mutations in the succinate dehydrogenase subunit sdhb cause susceptibility to familial pheochromocytoma and to familial paraganglioma. Am J Hum Genet 2001; 69: $49-54$.

9 Baysal BE, Rubinstein WS, Taschner PE: Phenotypic dichotomy in mitochondrial complex II genetic disorders. J Mol Med 2001; 79: $495-503$.

10 Gutman, M: Modulation of mitochondrial succinate dehydrogenase activity, mechanism and function. Mol Cell Biochem 1978; 20: 41-60.

11 Geromel V, Kadhom N, Ceballos-Picot I et al: Human cultured skin fibroblasts survive profound inherited ubiquinone depletion. Free Rad Res 2001, 35: 11-21.

12 Geromel V, Parfait B, von Kleist-Retzow JC et al: The consequences of a mild respiratory chain deficiency on substrate competitive oxidation in human mitochondria. Biochem Biophys Res Commun 1997; 236: 643-646.

13 Rustin P, Lance C: Succinate-driven reverse electron transport in the respiratory chain of plant mitochondria. The effects of rotenone and adenylates in relation to malate and oxaloacetate metabolism. Biochem J 1991; 274: 249-255.

14 Eaton S, Turnbull DM, Bartlett K: Redox control of betaoxidation in rat liver mitochondria. Eur J Biochem 1994; 220: $671-681$.

15 Rustin P, Parfait B, Chretien D et al: Fluxes of nicotinamide adenine dinucleotides through mitochondrial membranes in human cultured cells. J Biol Chem 1996; 271: 14785 - 14790.

16 Ernster L, Dallner G: Biochemical, physiological and medical aspects of ubiquinone function. Biochim Biophys Acta 1995; 1271: $195-204$.

17 Raha S, Robinson BH: Mitochondria, oxygen free radicals, disease and ageing. Trends Biochem Sci 2000; 25: 502-508.

18 Munnich A, Rustin P: Clinical spectrum and diagnosis of mitochondrial disorders. Am J Med Genet 2001; 106: 4-17.

19 Birch-Machin MA, Taylor RW, Cochran B, Ackrell BA, Turnbull DM: Late-onset optic atrophy, ataxia, and myopathy associated with a mutation of a complex II gene. Ann Neurol 2000; 48: 330 335.

20 Gimenez-Roqueplo AP, Favier J, Rustin P et al: The R22X mutation of the SDHD gene hereditary paraganglioma suppresses enzymatic activity of complex II in mitochondrial respiratory chain and induces activation of hypoxia pathway. Am J Hum Genet 2001; 69: 1186-1197.

21 Ishii N, Fujii M, Hartman PS et al: A mutation in succinate dehydrogenase cytochrome b causes oxidative stress and ageing in nematodes. Nature 1998; 394: 694-697. 